\title{
Utilizing Pharmacogenomics to Reduce Adverse Drug Events
}

\author{
Chandni Bardolia' ${ }^{1}$, Adriana Matos ${ }^{1}$, Veronique Michaud ${ }^{2}$, Jacques Turgeon ${ }^{2}$, Kevin T Bain ${ }^{1,3}$ \\ and Nishita S Amin ${ }^{1 *}$
}

${ }^{1}$ Tabula Rasa HealthCare, Applied Precision Pharmacotherapy Institute, USA

${ }^{2}$ Tabula Rasa HealthCare, Precision Pharmacotherapy Research and Development Institute, USA

${ }^{3}$ Biophilia LLC, USA

*Corresponding author: Nishita Amin, Tabula Rasa HealthCare, Applied Precision Pharmacotherapy Institute, Moorestown, New Jersey, USA.

To Cite This Article: Chandni Bardolia, Adriana Matos, Veronique Michaud, Jacques Turgeon, Kevin T Bain, Nishita S Amin, Utilizing Pharmacogenomics to Reduce Adverse Drug Events. Am J Biomed Sci \& Res. 2020 - 11(3). AJBSR.MS.ID.001638. DOI: 10.34297/AJBSR.2020.11.001638.

Received: 阱 November 19,2020; Published: 眥 December 22, 2020

\begin{abstract}
Objective: The objective of this publication is to describe how pharmacogenomic testing can help reduce the incidence of ADEs.

Summary: Pharmacogenomic testing identifies genetic variants that help clinicians determine an individual's expected response to medications. Results from pharmacogenomic testing may provide valuable information on the expected response to a drug while on the other hand, results may also help explain adverse drug events (ADEs), identify patients who may require closer monitoring to avoid ADEs, and avoid specific drugs that are expected to cause ADEs in certain individuals. Many ADEs are believed to have a genetic component. Drugs administered at normal doses may interact with a gene that has a genetic variant affecting the drug's response. Pharmacogenomic testing can identify genetic variants that encode specific CYP enzymes (e.g., CYP2C9, CYP2C19, CYP2D6, and CYP3As). It is estimated that drug-gene and drug-drug-gene interactions contribute to about one-third of potential major or substantial drug interactions that occur in patients. Pharmacogenomic testing can predict about 20 to 30 percent of ADEs and significantly reduce ADE associated deaths.
\end{abstract}

Conclusion: The field of pharmacogenomics is ever expanding, and evidence demonstrates that testing can help detect and prevent ADEs, including those with life-threatening clinical consequences. Given the tremendous financial humanistic costs associated with ADEs, healthcare providers should consider pharmacogenomic testing for their patients.

Keywords: Pharmacogenomics; Adverse Drug Events

Abbreviations: PGx-pharmacogenomic; ADEs-adverse drug events; FDA-Food and Drug Administration; DGI-drug-gene interaction; DDI-drugdrug interaction; DDGI-drug-drug-gene interaction; CYP-cytochrome; HSR-hypersensitivity reaction

\section{Introduction}

Pharmacogenomics (PGx) refers to a spectrum of genes that determine drug behavior and sensitivity [1]. PGx testing is used to predict how individual patients may respond to drugs based on their unique genetic profile [2]. Not only is PGx testing valuable in optimizing treatment efficacy, but the results can also aid in predicting and explaining adverse drug events (ADEs), identifying patients who may require closer monitoring to avoid ADEs, and avoiding specific drugs that are expected to cause
ADEs in certain individuals [3]. Currently, more than 200 drugs have PGx information on their U.S. Food and Drug Administration (FDA) approved labels [4]. This number will inevitably continue to grow as evidence surrounding the clinical impact of PGx becomes increasingly apparent.

The United States Department of Health and Human Services and the National Academy of Medicine define an ADE as "an injury resulting from medical intervention related to a drug" [5,6]. Based 
on this definition, an ADE encompasses adverse drug reactions, allergic reactions, overdoses, and medication errors [5]. ADEs are highly prevalent and costly but are often both predictable and preventable (up to 80 percent) [3]. Approximately five percent of all hospitalizations in the United States can be attributed to ADEs, and between 10 and 20 percent of patients experience ADEs while hospitalized [7]. The annual cost of ADEs is estimated to be $\$ 4$ billion in the United States [3]. Thus, it is no surprise that the FDA classifies ADEs as one of the major causes of morbidity and mortality and an important driver of healthcare costs [7-9]. While the majority of these statistics are reflective of the United States population, ADEs are pervasive worldwide, and older adults are more susceptible to ADEs [10].

Many ADEs are believed to have a genetic component [11]. A multitude of drugs interact with proteins coded from a gene that possesses non-synonymous genetic variants; the resulting variable expression of these proteins may affect a drug's response. This scenario is referred to as a drug-gene interaction (DGI), and it often goes unrecognized as a causative or contributive factor to ADEs [3,12]. A drug-drug-gene interaction (DDGI) involves a drug-drug interaction (DDI) superimposed on a DGI that can result in a process known as phenoconversion [12-14]. When phenoconversion occurs, a patient's genetically derived phenotype changes (e.g., normal metabolizer to poor metabolizer), which may also change the drug's response. It is estimated that DGIs and DDGIs contribute to about one-third of potential major or substantial drug interactions that occur in patients [14]. Evidence shows that the use of PGx test results can prevent about 20 to 30 percent of ADEs and considerably reduce ADE-associated deaths [9].

Many drugs are metabolized via the cytochrome P450 (CYP) enzymatic system [15]. PGx testing can identify genetic variants that encode specific CYP enzymes (e.g., CYP2C9, CYP2C19, and CYP2D6). Variants, or polymorphisms, in the CYP450 family of enzymes can significantly affect the pharmacokinetics and pharmacodynamics of medications and can perpetuate ADEs. For example, a study of patients taking clopidogrel found that those who are a CYP2C19 intermediate or poor metabolizer (individuals who express a reduced function variant of $C Y P 2 C 19$ ) or were concomitantly taking other drugs metabolized by this enzyme, had lower active metabolite levels, resulting in lower platelet inhibition. In patients taking clopidogrel who are rapid or ultra-rapid metabolizers (individuals who express an increased function variant of CYP2C19), higher active metabolite levels were noted, resulting in increased bleeding risk. It was determined that individuals with a non-normal metabolizer phenotype for the CYP2C19 enzyme or those who were concomitantly taking other drugs metabolized by this enzyme, required dosage adjustments in order to achieve a therapeutic response or to avoid ADEs with clopidogrel [16].
The effects of PGx testing has also been measured in patients taking abacavir $[17,18]$. The package insert for abacavir recommends that clinicians conduct $\mathrm{PGx}$ testing for variants of HLA-B and avoid prescribing abacavir to individuals who carry the $H L A-B^{*} 5701$ variant [19]. Clinical practice guidelines by the Clinical Pharmacogenetics Implementation Consortium (CPIC) [20] and the Dutch Pharmacogenetics Working Group (DPWG) also are available to help healthcare practitioners use PGx testing results to prevent hypersensitivity reactions (HSRs) with abacavir. A recently published study found that the incidence of abacavir-related HSRs decreased from 1.3 percent, on average, between 1999 and 2008 to 0.2 percent in 2015 due, in part, to genotyping for $H L A-B^{*} 5701$ [17]. The significance of this has been previously demonstrated, in which prospective PGx testing avoids 537 abacavir-related HSRs per 10,000 patients, while only costing an additional $\$ 17$ per patient [18]. These results suggest that prospective $H L A-B^{*} 5701$ screening, prior to abacavir initiation, avoids serious ADEs and produces cost savings.

While several factors affect patient response to warfarin, studies show that PGx testing may provide significant benefit [2123]. Knowledge about individual patient $C Y P 2 C 9$ gene variants, as well as other gene variants (i.e., VKORC1 and CYP4F2) can explain and, more importantly, help mitigate ADEs associated with warfarin use [21-23]. For example, genotype guided warfarin dosing in the Genetics Information Trial (GIFT) reduced the combined risk of major bleeding, International Normalized Ratio (INR) of 4 or greater, venous thromboembolism, or death [23]. Several evidencebased algorithms and dosing calculators use genetic information, in addition to other factors (e.g., gender, race, indication, smoker status), to guide warfarin dosing. While genetic information is important per se, phenoconversion and predictability of PGx results on warfarin effects may be altered in patients with polypharmacy due to DGI and DDGI [24]. A clinical practice guideline to help healthcare practitioners use PGx testing results to safely and accurately prescribe warfarin has been available since 2011, and was most recently updated in 2017 [25,26].

Though dosing guidelines and algorithms are publicly available for healthcare practitioners, PGx testing is woefully underused in clinical practice [27]. One of the contributing factors is that most clinical decision support systems (CDSS) do not have the capability of detecting DGIs, even when PGx test results are available $[13,28]$. In addition, testing is covered, in part or full, by some insurance providers, but coverage determinations vary substantially [29]. The out-of-pocket cost of PGx testing typically ranges from $\$ 200$ to $\$ 2,000$ ( $\$ 300$ average), depending on the genes included in the PGx test panel and technology used for testing [30]. A pivotal study conducted more than 20 years ago estimated the cost per ADE experienced in the inpatient setting to be $\$ 2,262$ [31]; a 2012 study 
indicated that ADEs cost more than $\$ 3,000$ and increases based on level of severity [32]. A recent study investigated the cost savings of PGx testing for depression and found that even at a cost of $\$ 2,000$, patients would save $\$ 3,962$ per year if $P G x$ tests were used to guide depression treatment [30]. Among 44 economic evaluations conducted of the FDA's Table of Pharmacogenomic Biomarkers in Drug Labeling, 57 percent drew conclusions that the use of PGx testing showed cost savings and/or cost effectiveness [33]. Other research shows that PGx testing for abacavir, carbamazepine, clopidogrel, and warfarin is cost effective, largely attributable to preventing significant ADEs, such as HSRs with abacavir and bleeding events from clopidogrel [3,34-36]. Lastly, a study that included 205 individuals, $\geq 65$ years of age with polypharmacy, showed that results obtained from PGx testing helped guide prescribing, which reduced hospitalization rates from 19.1 percent to 9.8 percent; this reduction completely offset the cost of the test and yielded net cost savings of $\$ 218$ per patient [37]. The potential cost savings of PGx testing is expected to increase over time given the one-time expense of testing and the potential to avoid lifetime ADEs.

In conclusion, researchers are constantly discovering genetic variants and their relation to drug response. The field of PGx is ever expanding, and evidence demonstrates that PGx testing can help detect and avoid ADEs, including life-threatening clinical consequences. Considering the potential cost savings associated with preventing ADEs, healthcare providers should consider PGX testing for their patients. Future research should continue to demonstrate cost savings associated with the use of PGx testing.

\section{Acknowledgement}

The authors want to thank Dana Filippoli and Amy Haines for their comprehensive review and comments on the content of this paper.

\section{Conflict of Interest statement}

All authors declare that there is no conflict of interest regarding the publication of this article. Dr.Bain is not an employee of Tabula Rasa HealthCare. All other authors are employees and shareholders of Tabula Rasa HealthCare.

\section{References}

1. Evans WE, Relling MV (1999) Pharmacogenomics: translating functional genomics into rational therapeutics. Science 286(5439): 487-491.

2. Hess GP, Fonseca E, Scott R, Fagerness J (2015) Pharmacogenomic and pharmacogenetic-guided therapy as a tool in precision medicine: current state and factors impacting acceptance by stakeholders. Genet Res (Camb) 97: e13.

3. Alfirevic A, Pirmohamed M (2017) Genomics of Adverse Drug Reactions. Trends Pharmacol Sci 38(1): 100-109.

4. Mehta D, Uber R, Ingle T, LiC, Liu Z, etal.(2020) Study of pharmacogenomic information in FDA approved drug labeling to facilitate application of precision medicine. Drug Discov Today 25(5): 813-820.
5. (2014) U.S. Department of Health and Human Services, Office of Disease Prevention and Health Promotion. National Action Plan for Adverse Drug Event Prevention. Washington, USA.

6. Institute of Medicine (2000) To Err Is Human: Building a Safer Health System. The National Academies Press, Washington, DC, 1-312, USA.

7. Schatz SN, Weber RJ (2015) Adverse drug reactions. CNS/Pharmacy Practice: 5-22.

8. Food and Drug Administration (2014) FDA adverse events reporting system (FAERS) patient outcomes by year.

9. Alagoz O, Durham D, Kasirajan K (2016) Cost-effectiveness of onetime genetic testing to minimize lifetime adverse drug reactions. Pharmacogenomics J 16(2): 129-136.

10. Cardelli M, Marchegiani F, Corsonello A, Lattanzio F, Provinciali M (2012) A review of pharmacogenetics of adverse drug reactions in elderly people. Drug Saf 35: 3-20.

11. Just KS, Dormann H, Schurig M, Bohme M, Fracowiak J, et al. (2020) Adverse Drug Reactions in the Emergency Department: Is There a Role for Pharmacogenomic Profiles at Risk? Results from the ADRED Study. J Clin Med 9(6): 1801.

12. Blagec K, Kuch W, Samwald M (2017) The Importance of Gene-DrugDrug-Interactions in Pharmacogenomics Decision Support: An Analysis Based on Austrian Claims Data. Stud Health Technol Inform 236: 121127.

13. Kim K, Magness JW, Nelson R, Baron V, Brixner DI (2018) Clinical Utility of Pharmacogenetic Testing and a Clinical Decision Support Tool to Enhance the Identification of Drug Therapy Problems Through Medication Therapy Management in Polypharmacy Patients. J Manag Care Spec Pharm 24(12): 1250-1259.

14. Verbeurgt P, Mamiya T, Oesterheld J (2014) How common are drug and gene interactions? Prevalence in a sample of 1143 patients with CYP2C9, CYP2C19 and CYP2D6 genotyping. Pharmacogenomics 15(5): 655-665.

15. Guengerich FP (1992) Characterization of human cytochrome P450 enzymes. FASEB J 6(2): 745-748.

16. Saab YB, Zeenny R, Ramadan WH (2015) Optimizing clopidogrel dose response: a new clinical algorithm comprising CYP2C19 pharmacogenetics and drug interactions. Ther Clin Risk Manag 11: 1421-1427.

17. Mounzer K, Hsu R, Fusco JS, Brunet L, Henegar CE, et al. (2019) HLA-B*57:01 screening and hypersensitivity reaction to abacavir between 1999 and 2016 in the OPERA observational database: a cohort study. AIDS Res Ther 16(1): 1.

18. Kauf TL, Farkouh RA, Earnshaw SR, Watson ME, Maroudas P, et al. (2010) Economic efficiency of genetic screening to inform the use of abacavir sulfate in the treatment of HIV. Pharmacoeconomics 28(11): 1025-1039.

19. (2012) Ziagen (Abacavir). In. Triangle Park, NC: Glaxo Smith Kline.

20. Martin MA, Hoffman JM, Freimuth RR, Klein TE, Dong BJ, et al. (2014) Clinical Pharmacogenetics Implementation Consortium Guidelines for HLA-B Genotype and Abacavir Dosing: 2014 update. Clin Pharmacol Ther 95(5): 499-500.

21. Mega JL, Walker JR, Ruff CT, Wandell AG, Nordio F, et al. (2015) Genetics and the clinical response to warfarin and edoxaban: findings from the randomised, double-blind ENGAGE AF-TIMI 48 trial. Lancet 385(9984): 2280-2287.

22. Pirmohamed M, Burnside G, Eriksson N, Jorgensen AL, Toh $\mathrm{CH}$, et al. (2013) A randomized trial of genotype-guided dosing of warfarin. N Engl J Med 369(24): 2294-2303.

23. Gage BF, Bass AR, Lin H, Woller SC, Stevens SM, et al. (2017) Effect of Genotype-Guided Warfarin Dosing on Clinical Events and Anticoagulation Control Among Patients Undergoing Hip or Knee Arthroplasty: The GIFT Randomized Clinical Trial. JAMA 318(12): 1115-1124. 
24. Michaud V, Vanier MC, Brouillette D, Roy D, Verret L, et al. (2008) Combination of phenotype assessments and CYP2C9-VKORC1 polymorphisms in the determination of warfarin dose requirements in heavily medicated patients. Clin Pharmacol Ther 83(5): 740-748.

25. Johnson JA, Gong L, Whirl Carrillo M, Gage BF, Scott SA, et al. (2011) Clinical Pharmacogenetics Implementation Consortium Guidelines for CYP2C9 and VKORC1 genotypes and warfarin dosing. Clin Pharmacol Ther 90(4): 625-629.

26. Johnson JA, Caudle KE, Gong L, Whirl-Carrillo M, Stein CM, et al (2017) Clinical Pharmacogenetics Implementation Consortium (CPIC) Guideline for Pharmacogenetics-Guided Warfarin Dosing: 2017 Update. Clin Pharmacol Ther 102(3): 397-404.

27. Zhou ZW, Chen XW, Sneed KB, Yang YX, Zhang X, et al. (2015) Clinica association between pharmacogenomics and adverse drug reactions. Drugs 75(6): 589-631.

28. Bain KT, Knowlton CH, Turgeon J (2017) Medication Risk Mitigation Coordinating and Collaborating with Health Care Systems, Universities, and Researchers to Facilitate the Design and Execution of Practice-Based Research. Clin Geriatr Med 33(2): 257-281.

29. Eichmeyer J, Rogers S, Formea CM, Giri J, Jones S, et al. (2020) PARC report: a perspective on the state of clinical pharmacogenomics testing. Pharmacogenomics 21(11): 809-820.

30. Maciel A, Cullors A, Lukowiak AA, Garces J (2018) Estimating cost savings of pharmacogenetic testing for depression in real-world clinical settings. Neuropsychiatr Dis Treat 14: 225-230.
31. Classen DC, Pestotnik SL, Evans RS, Lloyd JF, Burke JP (1997) Adverse drug events in hospitalized patients. Excess length of stay, extra costs, and attributable mortality. JAMA 277(4): 301-306.

32. Hug BL, Keohane C, Seger DL, Yoon C, Bates DW (2012) The costs of adverse drug events in community hospitals. Jt Comm J Qual Patient Saf 38(3): 120-126.

33. Verbelen M, Weale ME, Lewis CM (2017) Cost-effectiveness of pharmacogenetic-guided treatment: are we there yet? Pharmacogenomics J 17: 395-402.

34. Plumpton CO, Roberts D, Pirmohamed M, Hughes DA (2016) A Systematic Review of Economic Evaluations of Pharmacogenetic Testing for Prevention of Adverse Drug Reactions. Pharmacoeconomics 34(8): 771-793.

35. Johnson SG, Gruntowicz D, Chua T, Morlock RJ (2015) Financial Analysis of CYP2C19 Genotyping in Patients Receiving Dual Antiplatelet Therapy Following Acute Coronary Syndrome and Percutaneous Coronary Intervention. J Manag Care Spec Pharm 21(7): 552-557.

36. Verhoef TI, Redekop WK, Langenskiold S, Kamali F, Wadelius M, et al. (2016) Cost-effectiveness of pharmacogenetic-guided dosing of warfarin in the United Kingdom and Sweden. Pharmacogenomics J 16(5): 478-484

37. Brixner D, Biltaji E, Bress A, Unni S, Ye X, et al. (2016) The effect of pharmacogenetic profiling with a clinical decision support tool on healthcare resource utilization and estimated costs in the elderly exposed to polypharmacy. J Med Econ 19(3): 213-228. 